\title{
Impact of short-term mechanical circulatory support with extracorporeal devices on postoperative outcomes after emergency heart transplantation: Data from a multi-institutional Spanish cohort
}

\author{
Eduardo Barge-Caballero, Luis Almenar-Bonet, Adolfo Villa-Arranz, Félix Pérez-Villa, \\ Javier Segovia-Cubero, Juan Delgado-Jiménez, Francisco González-Vilchez, Nicolás \\ Manito-Lorite, Luis De-la-Fuente-Galán, Vicens Brossa-Loidi, Jose L. Lambert- \\ Rodríguez, Domingo Pascual-Figa, Ernesto Lage-Gallé, Jose M. Arizón-Del-Prado, \\ Marisa Sanz-Julve, Javier Muñiz-García, Marisa Crespo-Leiro
}

\begin{abstract}
Objectives. We sought to investigate the potential impact of preoperative short-term mechanical circulatory support (MCS) with extracorporeal devices on postoperative outcomes after emergency heart transplantation (HT).

Methods. We conducted an observational study of 669 patients who underwent emergency HT in 15 Spanish hospitals between 2000 and 2009. Postoperative outcomes of patients bridged to HT on short-term MCS $(\mathrm{n}=101)$ were compared with those of the rest of the cohort $(n=568)$. Short-term MCS included veno-arterial extracorporeal membrane oxygenators (VA-ECMOs, $n=23$ ), and both pulsatile-flow $(n=53)$ and continuous-flow $(n=25)$ extracorporeal ventricular assist devices (VADs). No patient underwent HT on intracorporeal VADs.

Results. Preoperative short-term MCS was independently associated with increased in-hospital postoperative mortality (adjusted odds-ratio 1.75, 95\% CI 1.05-2.91) and overall post-transplant mortality (adjusted hazard-ratio $1.60,95 \%$ CI 1.15-2.23). Rates of major surgical bleeding, cardiac reoperation, postoperative infection and primary graft failure were also significantly higher among MCS patients. Causes of death and survival after hospital discharge were similar in MCS and non-MCS candidates. Increased risk of post-transplant mortality affected patients bridged on pulsatile-flow extracorporeal VADs (adjusted hazard-ratio 2.21, 95\% CI 1.48-3.30) and continuous-flow extracorporeal VADs (adjusted hazard-ratio 2.24, 95\% CI 1.20-4.19), but not those bridged on VA-ECMO (adjusted hazard-ratio $0.51,95 \%$ CI $0.21-1.25$ ).

Conclusions. Patients bridged to emergency HT on short-term MCS are exposed to an increased risk of postoperative complications and mortality. In our series, preoperative bridging with VA-ECMO resulted in comparable posttransplant outcomes to those of patients transplanted on conventional support.
\end{abstract}

Keywords

Transplantation; Mechanical circulatory support; Extracorporeal

\section{Introduction}

The number of patients bridged to heart transplantation (HT) under mechanical circulatory support (MCS) is growing worldwide [1]. In this setting, the goal of MCS is not only to increase patient's life expectancy, but also to improve their quality of life, end-organ function and nutritional status, so favoring better posttransplant outcomes. However, the potential benefits of MCS may be counteracted by device-related complications [2] such as infection, embolism, bleeding, or immune sensitization.

Intracorporeal left ventricular assist devices (LVADs) constitute the current standard-of-care to provide long-term MCS to HT candidates whose clinical status deteriorates while waiting for an organ donor. According to the Interagency Registry for Mechanically Assisted Circulatory Support (INTERMACS), 1-year and 2-year survival rates of patients who currently receive a continuous-flow intracorporeal LVAD as a bridge-to-transplantation respectively exceed $80 \%$ and $70 \%$ [3]. In a pooled analysis of 12 observational studies, patients bridged to HT on intracorporeal LVADs showed comparable post-transplant survival to candidates bridged on optimal medical therapy, including intravenous inotropes [4]. A propensity-score-matched analysis of the United Network for Organ Sharing (UNOS) database reached a similar conclusion [5]. 
The implantation of an intracorporeal LVAD, however, is not the best therapeutic option for all HT candidates that require MCS. Postoperative mortality after LVAD surgery is significantly increased when the device is implanted in severely acute decompensated patients [6], or in the presence of right ventricular failure [7]. In most of these cases, the implantation of a short-term extracorporeal device constitutes a more suitable initial approach. Short-term MCS usually results in a rapid hemodynamic stabilization, and favors the recovery of the end-organ function, allowing the patient to undergo a destination procedure such as HT or long-term VAD implantation with a reasonable expectancy of survival [8]. Worldwide, the use of short-term extracorporeal devices as a direct bridge to HT is an uncommon strategy, as it implies that a suitable organ donor must become available for the patient within a few days. In Spain, however, it is frequently resorted to, as the use of long-term intracorporeal LVADs is subject to tight economic restrictions.

The potential impact of preoperative short-term extracorporeal MCS on post-transplant outcomes has been less studied than in the case of intracorporeal LVADs. Previous analyses included a small number of patients supported on short-term devices, and showed conflicting results. Some authors found that patients bridged to HT on extracorporeal MCS were exposed to an increased risk of post-transplant mortality [4] and [9], but others reported that they have similar post-transplant outcomes to candidates bridged on intravenous inotropes [10]. The potential impact of different extracorporeal devices on posttransplant outcomes has not yet been determined.

In view of gaps in knowledge, we sought to study the impact of preoperative short-term MCS on posttransplant outcomes. For this purpose, we analyzed in-hospital postoperative complications, long-term survival, and causes of death of patients who underwent emergency HT in Spain during the past decade after being supported with short-term extracorporeal VADs or veno-arterial extracorporeal membrane oxygenators (VA-ECMOs), and compared them with a control group of patients who underwent emergency HT without preoperative MCS during the same period.

\section{Methods}

\subsection{Setting of the study}

We conducted a multi-institutional, observational study based on a historical cohort of patients who underwent first, single-organ HT under a high-emergency status - the so-called status 0 - in the Spanish organ donor allocation system (Organización Nacional de Trasplantes, ONT) between January 1st, 2000 and December 31st, 2009.

During the study period, listing criteria for ONT status 0 required permanent hospitalization due to a non-reversible cardiac disease with an imminent risk of death, and either continuous dependence on shortterm extracorporeal MCS, an intra-aortic balloon pump (IABP), or invasive mechanical ventilation together with intravenous inotropes, or the presence of recurrent life-threatening ventricular arrhythmias despite optimal medical therapy. Heart transplant candidates listed under ONT status 0 had priority over all other candidates to get the first suitable donor heart available in the system. Long-term intracorporeal VADs were not available in Spain during the study period.

\subsection{Short-term mechanical circulatory support}

In this study, the definition of short-term MCS refers to extracorporeal devices intended for left ventricular, right ventricular, or biventricular circulatory assistance for a maximum period of a few weeks (usually < 30 days). This included continuous-flow extracorporeal VADs (Levitronix Centrimag, Medtronic Biomedicus, Medos HIA-VAD, Jostra Rotaflow and Impella 5L), pulsatile-flow extracorporeal VADs (Abiomed BVS5000, Abiomed AB5000, Berlin Heart Excor, and Thoratec), and VA-ECMO (Maquet PLS, Maquet Cardiohelp, Medos Deltastream, and Medtronic Biomedicus). Patients bridged to emergency HT solely under IABP support were included in the control group.

\subsection{Data collection and variables}

Data for the study were extracted from a multicentre database [11] that contains detailed clinical information about all consecutive patients aged > 18 years who underwent emergency HT between 2000 and 2009 in 15 out of the 16 Spanish hospitals that had an active HT program at that time. This database was assembled with data collected from the prospective Spanish National Heart Transplantation Registry [12] and completed on the basis of an individualized review of clinical records. For the present analysis, 
patients who underwent re-transplantation or double-organ transplantation were not considered. The institutional review board approved the study protocol.

\subsection{Post-transplant outcomes}

In-hospital postoperative outcomes of the study were major surgical bleeding, need for cardiac reoperation, need for dialysis, postoperative infection, primary graft failure, isolated right ventricular failure and in-hospital postoperative mortality. Long-term outcomes were post-transplant survival and discharge-conditioned post-transplant survival. Causes of death were collected from autopsy studies or, if not available, from medical certificates of death. Specific definitions of study outcomes have been detailed previously [11] and are provided as Supplementary data.

\subsection{Statistical analysis}

In this manuscript, categorical variables are presented as proportions and compared by means of the Chisquared test, while continuous variables are presented as mean \pm standard deviation and compared by means of the T-Student test.

Post-transplant survival curves of patients bridged to HT with or without short-term MCS were estimated by means of the Kaplan-Meier method and compared by means of the log-rank test, both in the entire cohort and in the subcohort of individuals who survived the early postoperative period and were discharged alive from hospital. Follow-up was censored at the time of death or the last clinical visit, up to a maximum of 5 years after HT.

Adjusted hazard-ratios (HRs) for overall post-transplant mortality and discharge-conditioned posttransplant mortality were obtained by means of backward stepwise multivariable Cox's proportional hazards models. Candidate variables that entered multivariable analyses were those that showed a statistically significant association $(\mathrm{p}<0.05)$ with each one of these outcomes in univariable analyses and others considered as potential confounders on the basis of previous literature and clinical experience. In a similar manner, the association between short-term MCS and in-hospital postoperative outcomes was assessed by means of backward stepwise multivariable logistic regression models.

In view of the asymmetric distribution of MCS and non-MCS patients over the study period, this was arbitrarily divided into two eras, the early era (years 2000 to 2006), and the recent era (years 2007 to 2009). Specific subanalyses about the impact of short-term MCS on post-transplant survival during both eras were conducted.

Statistical significance was set as a p-value $<0.05$, and all contrasts were two-tailed. Statistical analysis was performed with SPSS Statistics 20.

\section{Results}

\subsection{Study population}

According to the Spanish National Heart Transplantation Registry, 2956 patients aged > 18 years underwent HT in our country between 2000 and 2009. Seven hundred and twenty-four patients underwent HT under ONT status 0, with 711 of them at the 15 hospitals participating in the study. After the exclusion of 29 patients who underwent re-transplantation and 13 patients who underwent simultaneous double-organ transplantation, the final study sample comprised 669 patients.

One hundred and one $(15 \%)$ patients were bridged to HT on short-term MCS. Twenty-five patients from 9 centers were supported on continuous-flow extracorporeal VADs, 53 patients from 8 centers were supported on pulsatile-flow extracorporeal VADs, and 23 patients from 3 centers were supported on VAECMO. Extracorporeal VADs implanted included 43 univentricular devices (4 right VADs and 39 LVADs), and 35 biventricular devices. Among the 568 patients of the control group, $373(66 \%)$ were supported on an IABP before HT.

Fig. 1 shows the distribution of patients over the study period according to preoperative support. The proportion of patients undergoing HT under short-term MCS was 10.4\% during the period 2000-2003, $8.9 \%$ during the period 2004-2006 and 25.6\% during the period 2007-2009 (p for linear trend $<0.001$ ). In view of this asymmetric distribution, the study period was arbitrarily divided into two eras, the early era (years 2000 to 2006), and the recent era (years 2007 to 2009). The number (percentage) of MCS patients who underwent HT during the early and the recent era was, respectively, $43(43 \%)$ and $58(57 \%)$. 


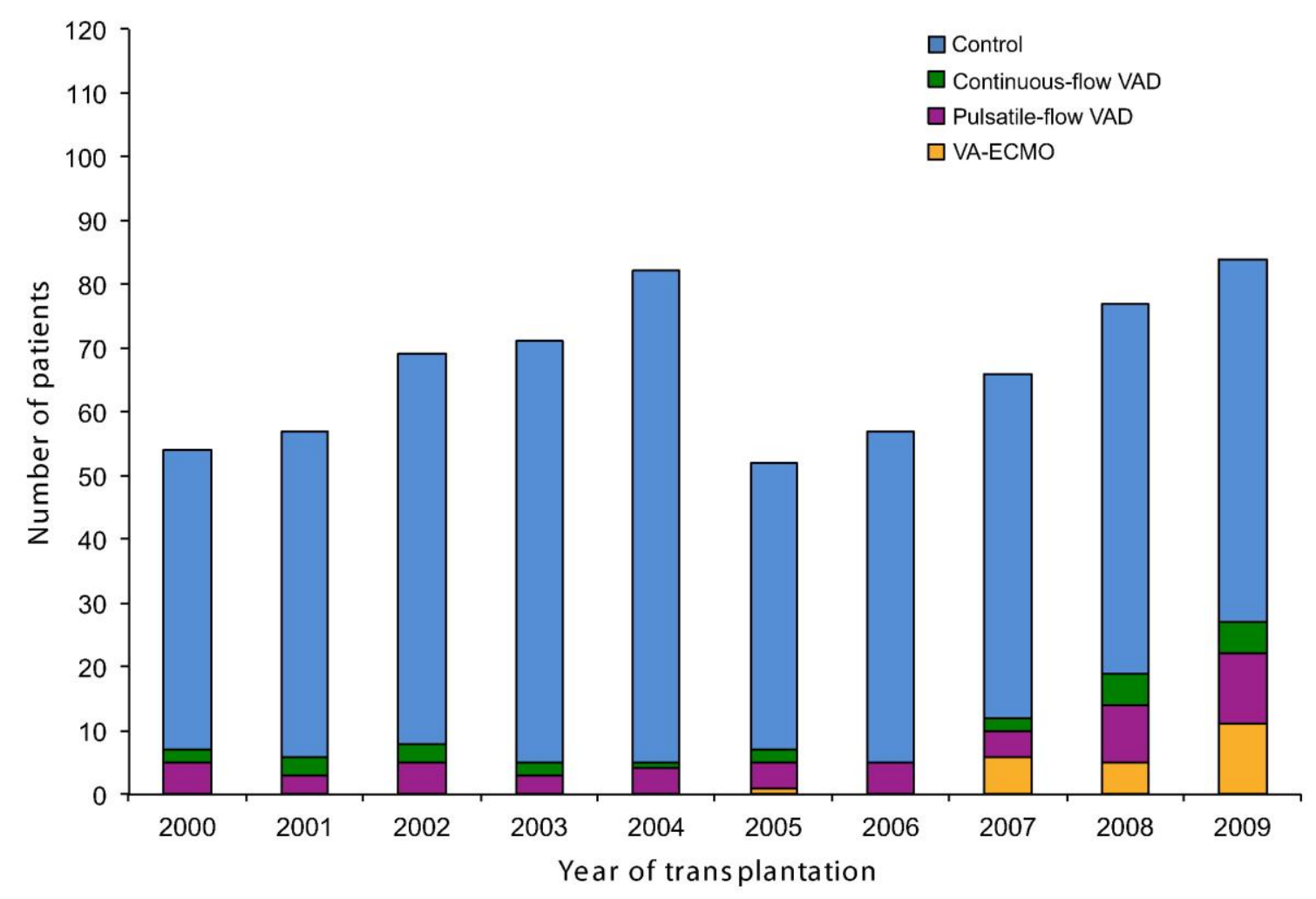

Fig. 1. Distribution of patients by the type of preoperative support and year of transplantation. VAD, ventricular assist device. VAECMO, veno-arterial extracorporeal membrane oxygenator.

\subsection{Clinical characteristics of patients with or without MCS}

Table 1 shows the clinical characteristics of recipients, donors, and HT procedures in the two study groups. Patients in the MCS group were younger and more frequently women than patients in the control group. The MCS group showed a higher prevalence of previous cardiac surgery and post-cardiotomy shock, while the prevalence of diabetes mellitus was higher in the control group. Mean waiting-list time for emergency HT was around 5 days in both study groups. 
Table 1. Clinical variables related to recipients, donors and heart transplant procedures.

\begin{tabular}{|c|c|c|c|}
\hline & $\begin{array}{c}\mathrm{MCS} \\
\mathrm{N}=101\end{array}$ & $\begin{array}{c}\text { Control } \\
\mathrm{N}=568\end{array}$ & $\mathrm{p}$ \\
\hline \multicolumn{4}{|l|}{ Demographics } \\
\hline Age (years), mean \pm standard deviation & $48 \pm 13$ & $50.9 \pm 12$ & 0.022 \\
\hline Women & $38 \%$ & $16 \%$ & $<0.001$ \\
\hline Waiting-list time (days) & $5.8 \pm 7$ & $5.2 \pm 7$ & 0.42 \\
\hline \multicolumn{4}{|l|}{ Clinical history } \\
\hline Ischemic heart disease & $44 \%$ & $49 \%$ & 0.30 \\
\hline Acute myocardial infarction & $35 \%$ & $32 \%$ & 0.66 \\
\hline Diabetes & $6 \%$ & $16 \%$ & 0.011 \\
\hline Hypertension & $23 \%$ & $27 \%$ & 0.35 \\
\hline Previous cardiac surgery & $50 \%$ & $17 \%$ & $<0.001$ \\
\hline Post-cardiotomy shock & $39 \%$ & $16 \%$ & $<0.001$ \\
\hline Implantable defibrillator & $12 \%$ & $23 \%$ & 0.012 \\
\hline Cardiac resynchronization therapy & $3 \%$ & $8 \%$ & 0.083 \\
\hline Cardiac arrest & $20 \%$ & $20 \%$ & 0.92 \\
\hline Preoperative infection & $35 \%$ & $27 \%$ & 0.12 \\
\hline Preoperative INTERMACS status & & & $<0.001$ \\
\hline Status 1 & $39 \%$ & $28 \%$ & \\
\hline Status 2 & $50 \%$ & $39 \%$ & \\
\hline Status 3 & $11 \%$ & $28 \%$ & \\
\hline Status 4 & $1 \%$ & $5 \%$ & \\
\hline \multicolumn{4}{|l|}{ Supportive therapies } \\
\hline Intravenous inotropes & $94 \%$ & $94 \%$ & 0.88 \\
\hline Inotropic index ${ }^{a}$ & $63 \pm 82$ & $23 \pm 46$ & $<0.001$ \\
\hline Dialysis & $9 \%$ & $7 \%$ & 0.60 \\
\hline Mechanical ventilation & $87 \%$ & $38 \%$ & $<0.001$ \\
\hline \multicolumn{4}{|l|}{ Laboratory } \\
\hline Hemoglobin $(\mathrm{g} / \mathrm{l})$, at transplantation & $10.2 \pm 2.5$ & $11.5 \pm 2$ & $<0.001$ \\
\hline Creatinine $(\mathrm{mg} / \mathrm{dl})$, peak & $1.65 \pm 0.8$ & $1.63 \pm 0.9$ & 0.82 \\
\hline Creatinine $(\mathrm{mg} / \mathrm{dl})$, at transplantation & $1.35 \pm 0.7$ & $1.32 \pm 0.7$ & 0.90 \\
\hline Bilirubin $(\mathrm{mg} / \mathrm{dl})$, peak & $2.4 \pm 2.1$ & $1.9 \pm 1.6$ & 0.047 \\
\hline Bilirubin $(\mathrm{mg} / \mathrm{dl})$, at transplantation & $1.64 \pm 1.4$ & $1.63 \pm 1.5$ & 0.97 \\
\hline Aspartate aminotransferase (IU/l), peak & $482 \pm 1249$ & $207 \pm 631$ & 0.038 \\
\hline Aspartate aminotransferase (IU/l), at transplantation & $182 \pm 552$ & $154 \pm 574$ & 0.65 \\
\hline Alanine aminotransferase (IU/l), peak & $389 \pm 1032$ & $194 \pm 622$ & 0.013 \\
\hline Alanine aminotransferase (IU/l), at transplantation & $157 \pm 471$ & $147 \pm 551$ & 0.87 \\
\hline \multicolumn{4}{|l|}{ Hemodynamics } \\
\hline Ejection fraction $(\%)$ & $19 \pm 11$ & $21 \pm 9$ & 0.022 \\
\hline Cardiac index $\left(\mathrm{ml} / \mathrm{min} / \mathrm{m}^{2}\right)$ & $2.1 \pm 0.7$ & $2.2 \pm 0.7$ & 0.32 \\
\hline Central venous pressure (mm Hg) & $14 \pm 6$ & $13 \pm 7$ & 0.25 \\
\hline Capillary wedge pressure (mm Hg) & $23 \pm 9$ & $25 \pm 9$ & 0.13 \\
\hline Mean pulmonary pressure $(\mathrm{mm} \mathrm{Hg})$ & $32 \pm 10$ & $33 \pm 10$ & 0.23 \\
\hline \multicolumn{4}{|l|}{ Heart transplant surgery } \\
\hline Cold ischemic time (min) & $219 \pm 58$ & $211 \pm 58$ & 0.24 \\
\hline Bypass time (min) & $160 \pm 73$ & $131 \pm 50$ & $<0.001$ \\
\hline \multicolumn{4}{|l|}{ Donor characteristics } \\
\hline Female donor & $35 \%$ & $27 \%$ & 0.10 \\
\hline Donor on inotropes & $78 \%$ & $77 \%$ & 0.76 \\
\hline Donor with cardiac arrest & $6 \%$ & $8 \%$ & 0.69 \\
\hline Donor age (years) & $36.3 \pm 14$ & $36 \pm 13$ & 0.82 \\
\hline
\end{tabular}

INTERMACS, Interagency Registry for Mechanically Assisted Circulatory Support. UI, international units. MCS, mechanical circulatory support.

a

Inotropic index [19] was calculated as dopamine dose $(\mu \mathrm{g} / \mathrm{kg} / \mathrm{min})+$ dobutamine dose $(\mu \mathrm{g} / \mathrm{kg} / \mathrm{min})+10 \times$ milrinone dose $(\mu \mathrm{g} / \mathrm{kg} / \mathrm{min})+100 \times$ epinephrine dose $(\mu \mathrm{g} / \mathrm{kg} / \mathrm{min})+100 \times$ norepinephrine dose $(\mu \mathrm{g} / \mathrm{kg} / \mathrm{min})$. 
Mechanically supported patients required higher doses of intravenous inotropes and were more frequently on ventilator before HT. Patients in the MCS group reached significantly higher peak serum levels of bilirubin, aspartate aminotransferase and alanine aminotransferase during the pre-transplant hospitalization, but levels were similar in both groups at the time of HT. Peak serum creatinine and creatinine at the time of HT were also similar in both study groups. The proportion of patients with INTERMACS status 1, 2, 3 and 4 before HT was respectively $39 \%, 50 \%, 11 \%$ and $1 \%$ in the MCS group and $28 \%, 39 \%, 28 \%$ and $5 \%$ in the control group.

Mean bypass time during HT surgery was significantly longer $(\mathrm{p}<0.001)$ in the MCS group $(160 \pm 73 \mathrm{~min})$ than in the control group $(131 \pm 50 \mathrm{~min})$. No significant differences among groups were observed with regard to donor age or cold ischemic times.

\subsection{In-hospital postoperative outcomes}

During the early postoperative period after HT, patients bridged under short-term MCS presented significantly higher rates of primary graft failure $(36.6 \%$ versus $21.3 \%, p=0.001)$, major surgical bleeding $(30.7 \%$ versus $21.5 \%, p=0.042)$, need for cardiac reoperation $(21.8 \%$ versus $13.2 \%, p=0.024)$, and postoperative infection $(50.5 \%$ versus $38.6 \%, \mathrm{p}=0.024)$ than patients in the control group, as shown in Fig. 2. Rates of isolated ventricular failure and postoperative dialysis were similar in both groups.

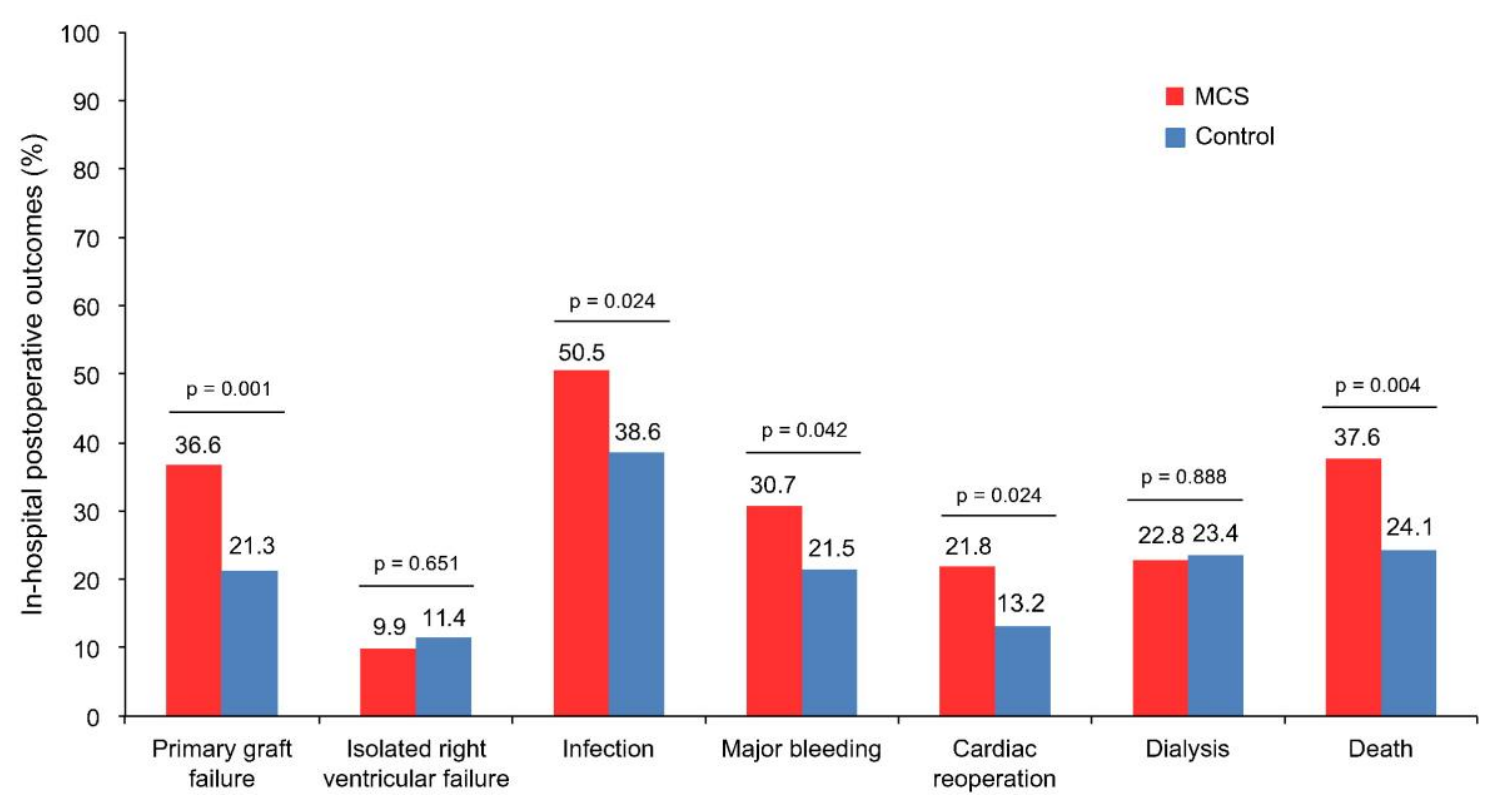

Fig. 2. Rates of in-hospital postoperative outcomes in patients with or without pre-transplant short-term MCS. MCS, mechanical circulatory support.

By means of multivariable logistic regression, preoperative short-term MCS was identified as an independent risk factor for primary graft failure (adjusted odds-ratio (OR) 1.78, 95\% confidence interval (CI) 1.08-2.94) and cardiac reoperation (adjusted OR 1.93, 95\% CI 1.12-3.35). A non-significant trend toward increased risk of major surgical bleeding was also observed in MCS patients (adjusted OR 1.55, 95\% CI 0.97-2.50). The association between short-term MCS and postoperative infection lost its statistical significance after multivariable adjustment (adjusted OR 1.04, 95\% CI 0.65-1.67). 


\subsection{Post-transplant survival}

Median post-transplant follow-up was 2.9 years (interquartile rank, 0.2 to 5 years). Overall, 255 (38\%) patients died, 175 (26\%) during in-hospital follow-up and 80 (12\%) after hospital discharge.

Patients in the MCS group had significantly shorter post-transplant survival than patients in the control group $(\log$ rank $\mathrm{p}=0.004$, Fig. 3, panel A). By means of multivariable Cox's proportional hazards regression (Table 2), preoperative short-term MCS was identified as an independent risk factor for overall post-transplant mortality (adjusted hazard-ratio (HR) 1.60, 95\% CI 1.15-2.23).

A

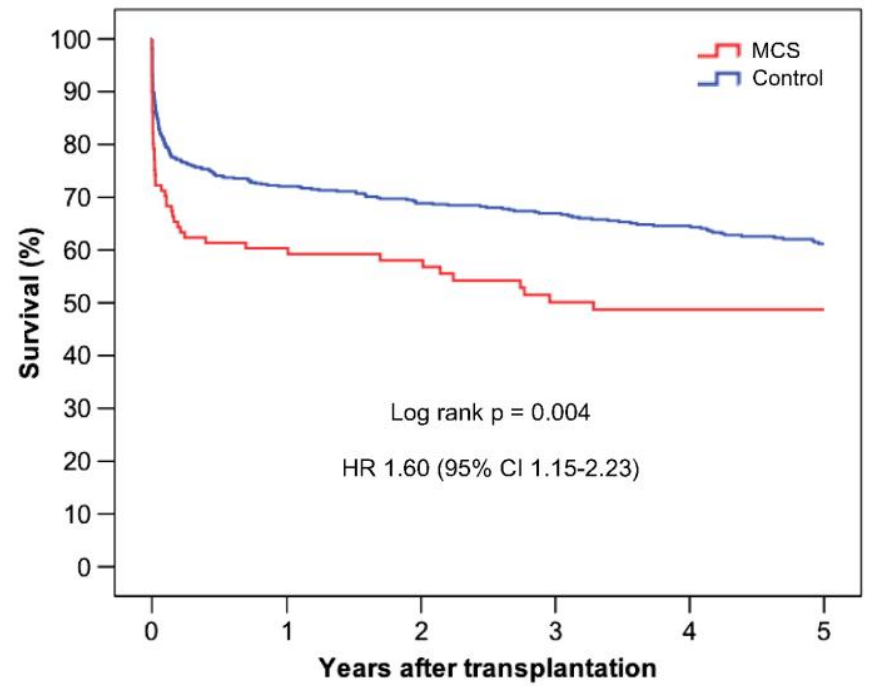

Pa tients at risk $(n)$

\begin{tabular}{lcccccc}
\hline MCS & 101 & 55 & 46 & 36 & 30 & 20 \\
Control & 568 & 384 & 330 & 295 & 261 & 220
\end{tabular}

B

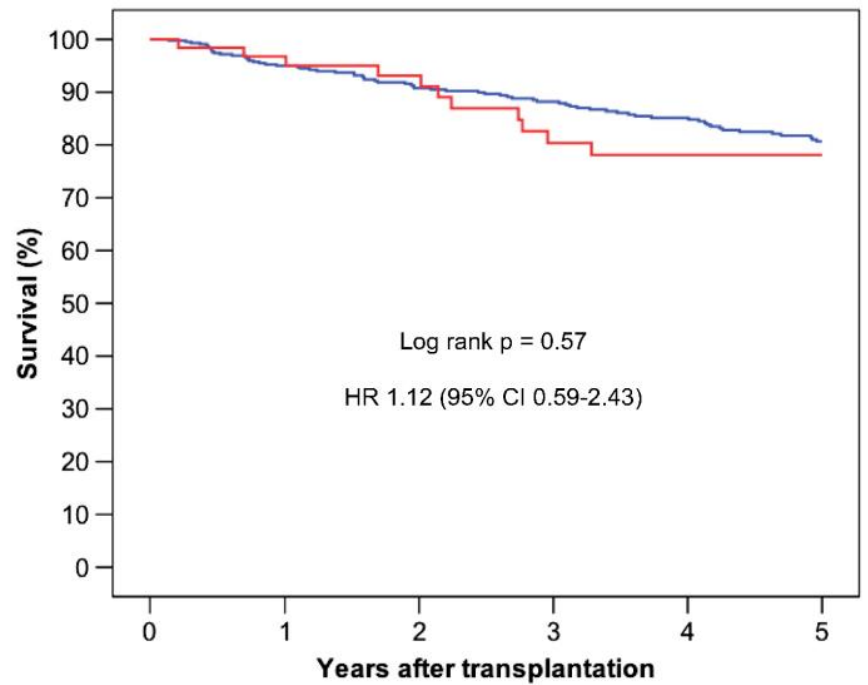

Pa tients at risk (n)

$\begin{array}{lrrrrrr}\text { MCS } & 62 & 55 & 46 & 36 & 30 & 20 \\ \text { Control } & 430 & 384 & 330 & 295 & 261 & 220\end{array}$

Fig. 3. Post-transplant survival of patients with or without preoperative short-term MCS. Panel A, overall post-transplant survival. Panel B, discharge-conditioned post-transplant survival. CI, confidence interval. HR, hazard ratio. MCS, mechanical circulatory support. 
Table 2. Risk factors for post-transplant mortality: multivariable Cox's proportional hazards regression.

\begin{tabular}{lll}
\hline & \multicolumn{1}{c}{ Adjusted HR (95\% CI) } & $\mathrm{p}$ \\
\hline & & \\
Age of the recipient (years) & $1.012(1-1.024)$ & 0.049 \\
Age of the donor (years) & $1.014(1.003-1.024)$ & 0.010 \\
Diabetes mellitus & $1.51(1.06-2.16)$ & 0.022 \\
Ischemic heart disease & $0.70(0.53-0.92)$ & 0.011 \\
Pre-transplant dialysis & $2.15(1.46-3.18)$ & $<0.001$ \\
Pre-transplant short-term MCS & $1.60(1.15-2.23)$ & 0.006 \\
& & \\
\hline
\end{tabular}

CI, confidence interval. HR, hazard ratio. MCS, mechanical circulatory support.

In-hospital postoperative mortality was significantly higher in the MCS group than in the control group (37.6\% versus $24.1 \%, \mathrm{p}=0.004$; adjusted OR $1.75,95 \%$ CI 1.05-2.91, Fig. 2). Preoperative shortterm MCS had no significant impact on discharge-conditioned post-transplant survival ( $\log$ rank $\mathrm{p}=0.57$; adjusted HR 1.19, 95\% CI 0.59-2.43, Fig. 3, panel B).

\subsection{Causes of death}

Primary graft failure, infection and non-specified multi-organ failure were the most frequent causes of death during the early postoperative period, while rejection (acute and chronic), malignancy and infection accounted for the majority of deaths during post-discharge follow-up. Fig. 4 shows the distribution of the causes of death in the two study groups. 


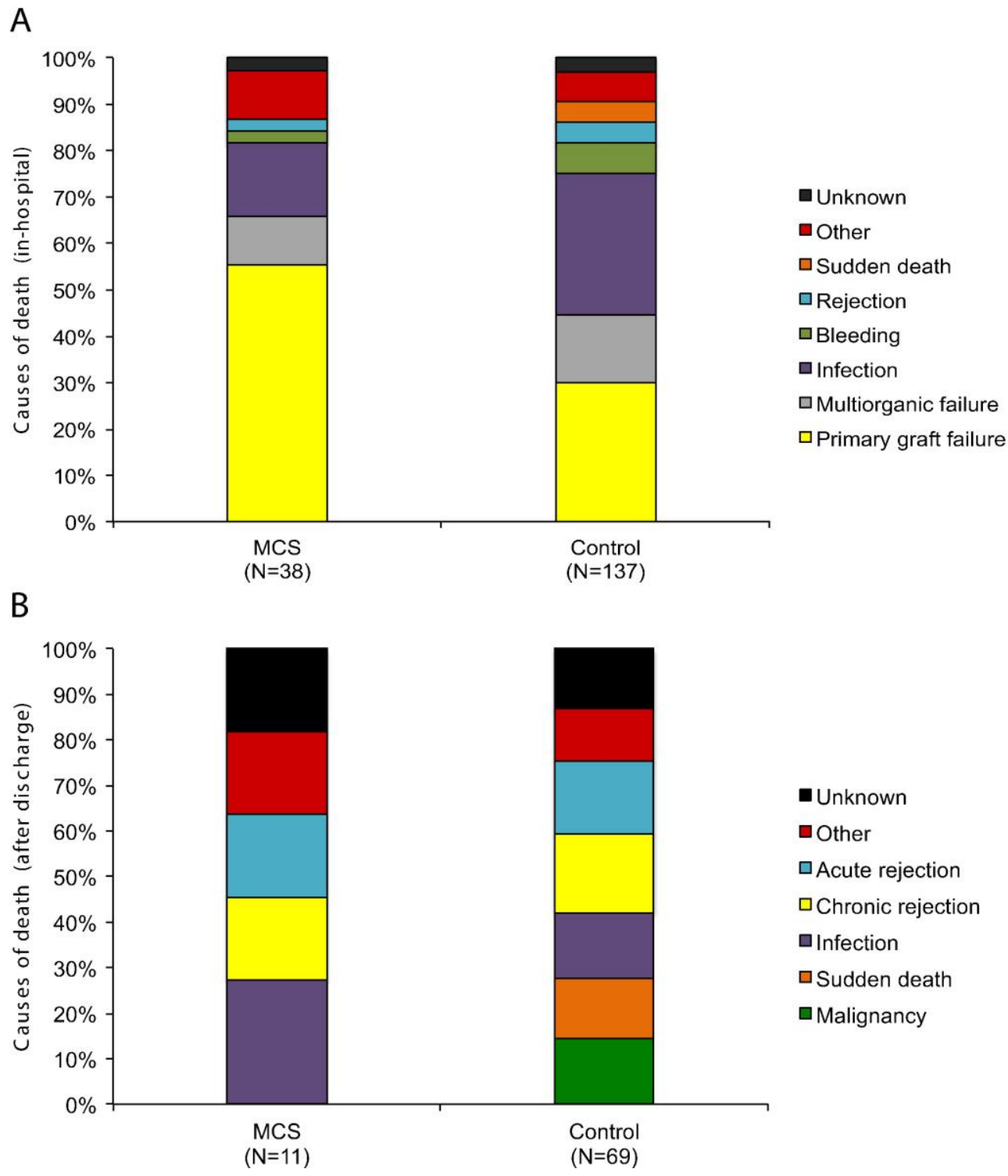

Fig. 4. Causes of death in the study groups. Panel A, causes of death during the in-hospital postoperative period. Panel B, causes of death after hospital discharge. MCS, mechanical circulatory support.

\subsection{Transplant eras}

As shown in Fig. 5, short-term MCS was associated with increased post-transplant mortality only among patients who underwent HT during the early era $(\log$ rank $\mathrm{p}=0.006$; adjusted HR 2.14, 95\% CI 1.323.46), but not among those who underwent HT during the recent era ( $\log$ rank $\mathrm{p}=0.41$, adjusted HR 1.06 , 95\% CI 0.66-1.69). In comparison to the early era, the recent era was characterized by significantly longer mean waiting-list times (6.1 versus 4.8 days, $\mathrm{p}=0.033)$ and mean cold ischemic times $(224$ versus $206 \mathrm{~min}, \mathrm{p}<0.001$ ), and also by the use of older donors (mean age 38.5 versus 34.7 years, $\mathrm{p}<0.001$ ) with a higher prevalence of previous cardiac arrest $(11.2 \%$ versus $5.2 \%, \mathrm{p}=0.011)$. 
A

Ea rly era (2000-2006)

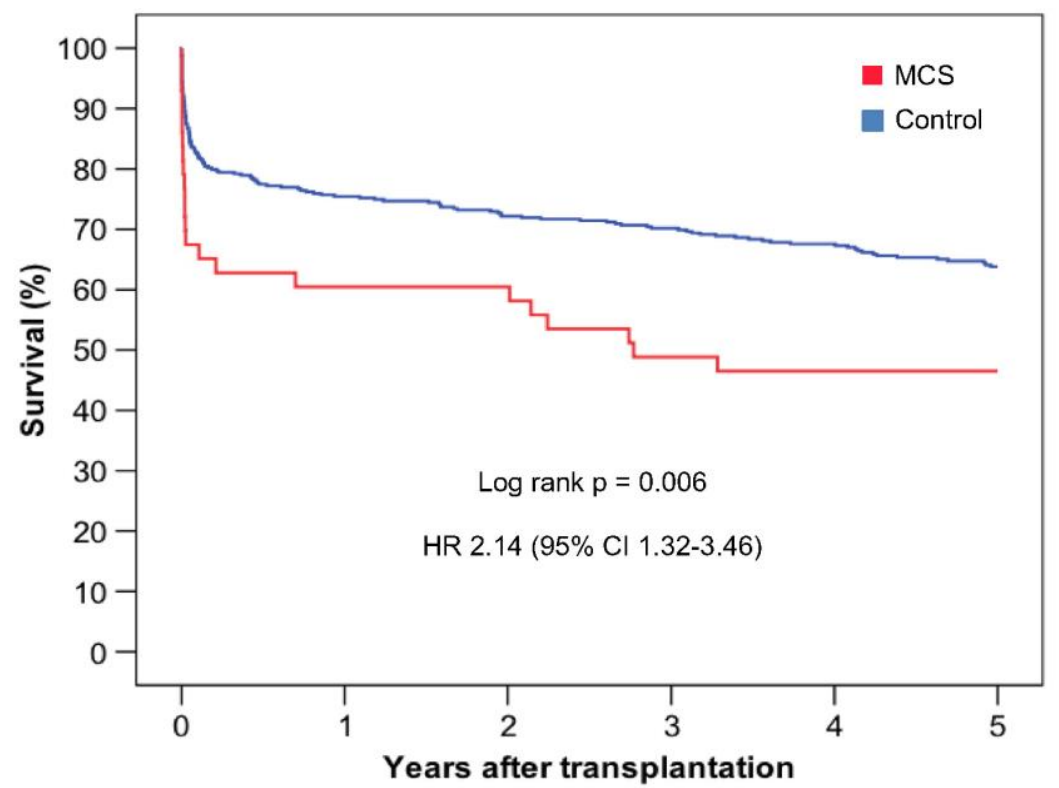

Pa tients at risk (n)

\begin{tabular}{lrrrrrr}
\hline MCS & 43 & 26 & 26 & 21 & 18 & 16 \\
Control & 399 & 301 & 288 & 276 & 246 & 208
\end{tabular}

B

Recent era (2007-2009)

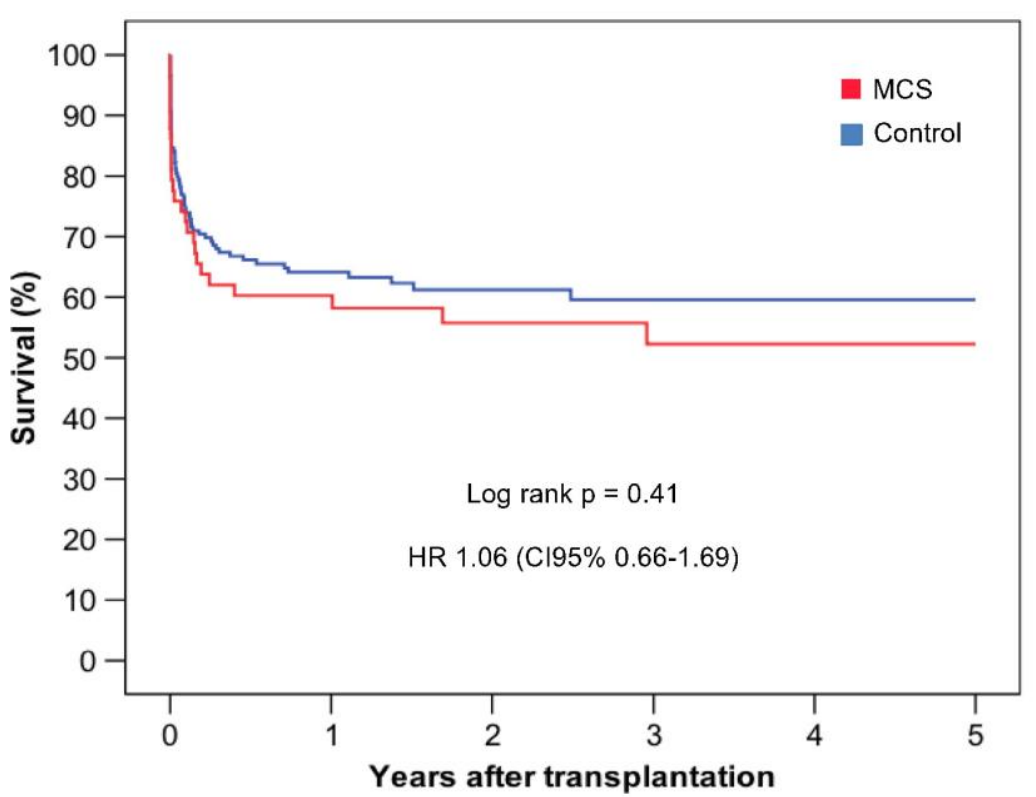

Pa tients at risk (n)

$\begin{array}{lcccccc}\text { MCS } & 58 & 29 & 20 & 14 & 11 & 4 \\ \text { Control } & 169 & 83 & 42 & 19 & 15 & 12\end{array}$

Fig. 5. Long-term post-transplant survival of patients with or without preoperative short-term MCS according to transplant eras. Panel A, early era (years 2000-2006). Panel B, recent era (years 2007-2009). CI, confidence interval. HR, hazard ratio. MCS, mechanical circulatory support. 


\subsection{Impact of different devices on post-transplant survival}

Fig. 6 shows the Kaplan-Meier post-transplant survival curves in patients bridged to HT on continuousflow extracorporeal VADs, pulsatile-flow extracorporeal VADs, and VA-ECMO $(\log \operatorname{rank} \mathrm{p}<0.001)$. With regard to the control group, post-transplant mortality was significantly increased among patients supported on pulsatile-flow extracorporeal VADs (adjusted HR 2.21, 95\% CI 1.48-3.30) and continuousflow extracorporeal VADs (adjusted HR 2.24, 95\% CI 1.20-4.19), but not among those supported on VAECMO (adjusted HR 0.51, 95\% CI 0.21-1.25). Both univentricular extracorporeal VADs (adjusted HR 2.08, 95\% CI 1.33-3.24) and biventricular extracorporeal VADs (adjusted HR 2.44, 95\% CI 1.46-4.06) were associated with increased post-transplant mortality.

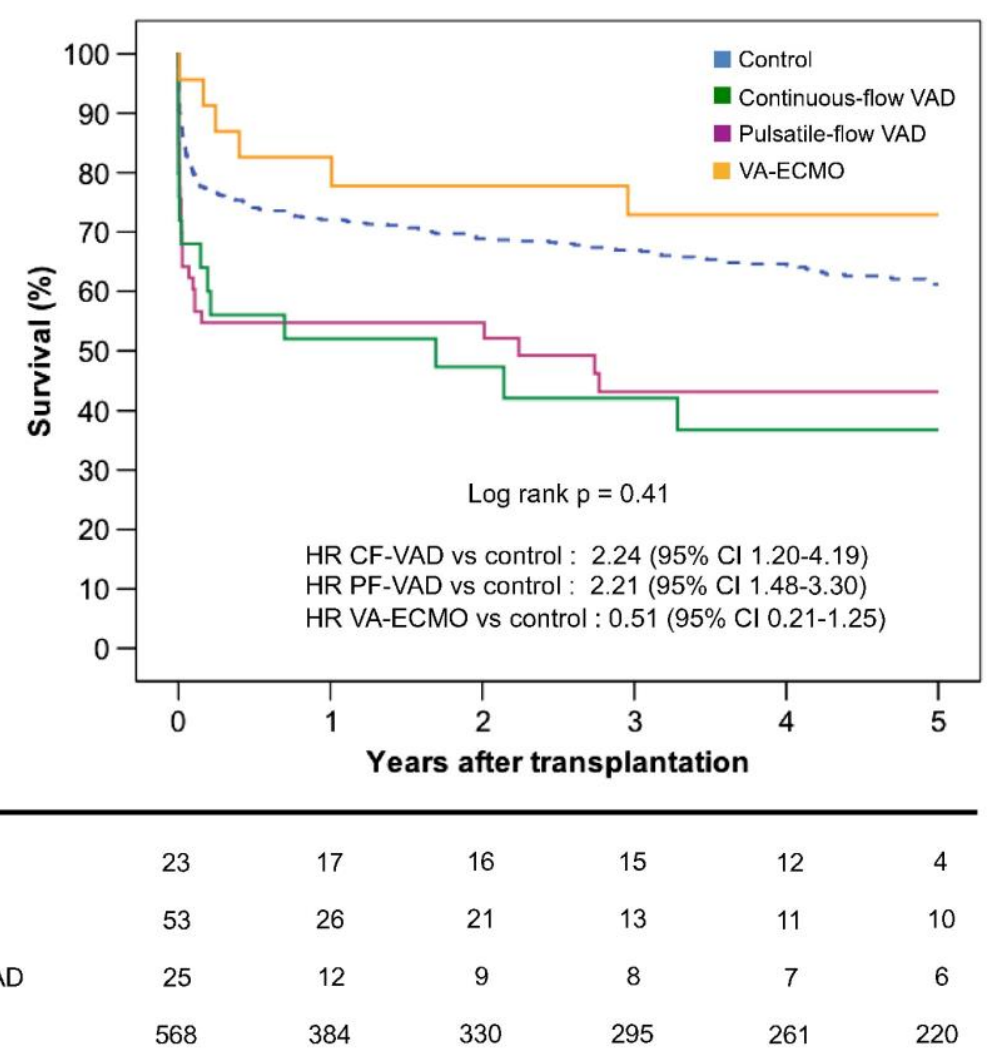

Fig. 6. Long-term post-transplant survival with regard to the type of preoperative support. CF, continuous-flow. CI, confidence interval. HR, hazard ratio. PF, pulsatile-flow. VAD, ventricular assist device. VA-ECMO, veno-arterial extracorporeal membrane oxygenator.

Baseline characteristics of patients bridged to HT on VA-ECMO were similar to those of patients bridged on extracorporeal VADs, with the exception that former individuals were older (mean age VAECMO group, 54.1 \pm 10 years; extracorporeal VAD group, $46.2 \pm 13$ years; $p=0.008$ ). At transplant surgery, patients bridged on VA-ECMO presented shorter mean cold ischemic times (194 \pm 57 versus $226 \pm 57 \mathrm{~min}, \mathrm{p}=0.022)$ and mean bypass times $(139 \pm 43$ versus $167 \pm 79, \mathrm{p}=0.031)$ than patients bridged on extracorporeal VADs. 


\section{Discussion}

In our retrospective analysis of a multi-institutional cohort of Spanish patients who underwent emergency HT from 2000 to 2009, we found that candidates bridged on short-term MCS with extracorporeal devices had shorter post-transplant survival than those bridged with conventional management, which included intravenous inotropes, IABP and mechanical ventilation as clinically required. By means of multivariable models, we estimated an adjusted hazard-ratio for post-transplant mortality of 1.60 (95\% CI 1.15-2.23) for MCS patients. To a large extent, this result was driven by an increased incidence of postoperative complications and death during the early in-hospital period after HT. Increased mortality risk affected patients bridged to HT on pulsatile-flow and continuous-flow extracorporeal VADs, but not those bridged on VA-ECMO. Causes of death and long-term survival after hospital discharge were similar in MCS and non-MCS patients.

From clinical experience, surgeons have learnt that HT procedures in MCS patients may be even more challenging and bloody than usual. Many devices involve the need for an additional sternotomy and require chronic antithrombotic therapy until the day of transplantation. Excessive bleeding during HT surgery may lead to prolonged cold ischemic and bypass times, and frequently involve the need for early redo cardiac procedures. In this clinical setting, the viability of the vulnerable donor heart may be compromised, increasing the risk of primary graft failure and subsequent associated complications, like nosocomial infection, multi-organ dysfunction, and death. This reality is well reflected in our study, even though mean pre-transplant waiting-list times were really short in both study groups - around 5 days and that no significant differences were noticed between MCS and non-MCS patients with regard to the quality of selected donors.

In general, our findings are concordant with previous literature. A pooled analysis of two observational studies [13] and [14] including 461 MCS patients and 9475 controls showed that candidates bridged to high-priority HT on short-term extracorporeal VADs had significantly increased posttransplant mortality, with a relative risk of 1.8 (95\% CI 1.5-2.1). Extracorporeal MCS was also identified as an independent risk factor for post-transplant mortality in the multi-institutional registries of the International Society for Heart and Lung Transplantation [1] and the UNOS [9]. A recent Spanish singlecenter study [10], however, reported similar post-transplant outcomes for patients bridged to emergency HT with or without short-term MCS in a particular setting of short waiting-list times. Surgical risks associated to MCS appear to have a less relevant prognostic impact in the case of stable patients undergoing HT on long-term intracorporeal VADs [4] and [5], possibly because they are compensated for by the beneficial effects of the device on patient's functional status, nutrition or end-organ function.

The results of the present investigation should be interpreted with caution, because - similar to previous studies - the baseline clinical characteristics of MCS and non-MCS HT candidates were not exactly the same. Mechanically supported patients were younger than controls, but the former presented with a more severely compromised clinical condition during the pre-transplant hospitalization - higher inotropic requirements, more frequent need for mechanical ventilation, larger extent of liver damage that justified the implantation of a short-term device. At the time of HT, however, end-organ function and hemodynamic parameters were similar in both study groups, reflecting that MCS was reasonably effective in those critically ill individuals.

Our analysis is affected by a significant era effect. During the earlier years of the study (2000-2006), post-transplant mortality was significantly higher among MCS patients than among controls, but this difference disappeared during the most recent era (2007-2009). In part, this finding might be attributable to a "learning curve" effect, as the results of MCS programs tend to improve with increasing experience. Survival differences across eras may also be affected by the use of different mechanical devices. As discussed later, post-transplant survival was significantly better among patients bridged on VA-ECMO, which were mostly implanted in the recent era, than among those bridged on continuous-flow and pulsatile flow devices, which were mainly used in the early era. Finally, it should be noticed that the tendency to a similar post-transplant survival in MCS and non-MCS patients observed in the recent era is strongly conditioned by a significant increase of post-transplant mortality in the latter group in comparison with previous years. In our opinion, this reality reflects a new scenario of increasing scarcity of donors, which has made HT teams more willing to accept less-adequate organs from older donors and exposed to longer cold ischemic times.

In our series, post-transplant survival of MCS patients varied significantly according to the type of device implanted. Candidates supported on pulsatile-flow and continuous-flow extracorporeal VADs showed the highest post-transplant mortality, independently of whether they received univentricular or biventricular support. Preoperative bridging with VA-ECMO, however, did not carry a significant increase of post-transplant mortality in comparison with the control group. This finding must also be interpreted with caution, as the use of VA-ECMO was limited to a small number of patients and centers in our series. 
Some advantages make VA-ECMO an attractive option for the initial management of HT candidates presenting with a critical clinical condition. Full circulatory and pulmonary support through a peripheral vascular access may be provided quickly, at the bedside, and avoiding additional sternotomies. Different to other devices, VA-ECMO carries a low risk of thrombotic complications, so patients may be safely managed with low-dose unfractionated heparin, without concomitant antiplatelet therapy. To minimize bleeding risk, a normal coagulation state may be easily restored just before HT surgery by administering intravenous protamine.

The use of VA-ECMO as a bridge to emergency HT in cardiogenic shock patients offers satisfactory results in systems characterized by short waiting-list times [15], as is the case of the Spanish ONT. Indeed, VA-ECMO is currently the most frequent device implanted with a "bridge-to-transplant" indication in our country [12]. However, the feasibility of this strategy is compromised by the limited durability of the device, usually not longer than 2 to 3 weeks. The need for prolonged VA-ECMO support, indeed, is associated with a high risk of device-related complications and worsening posttransplant outcomes [16] and [17]. This implies that, in case the patient does not get a suitable organ donor in a short lapse of time, a switch to a durable device must be considered. Until now, the efficacy of this "bridge-to-bridge" strategy remains controversial as, in patients under temporary MCS, the implantation of an intracorporeal LVAD also carries a high mortality risk [18].

The major limitation of our study is its observational, non-randomized design, which implies that its usefulness is limited to hypothesis generation. As pointed out, study findings are conditioned by significant differences in the baseline clinical characteristics of MCS and non-MCS patients. Despite an extensive multivariable adjustment being conducted in order to minimize confusion bias, we cannot rule out that other non-tested variables may have influenced the observed statistical associations. Similar to previous studies, our analysis is limited to post-transplant outcomes, so no conclusion may be extracted about a potential positive impact of MCS on pre-transplant survival. Given the period studied (2000 to 2009), our investigation reflects a past reality in the rapidly evolving field of MCS and HT, so its results might not be extrapolated to the current era. In view of the progressive development of more simple and safer mechanical devices and the continuous refinement of organ donor allocation policies, a significant improvement of the results of emergency HT in MCS patients is expectable in the future years. Finally, because of the peculiarities of the Spanish national organ donor allocation system, the external validity of the study is not warranted, so our conclusions might not be applicable to other countries.

In conclusion, our study supports the hypothesis that patients undergoing emergency HT on shortterm MCS with extracorporeal devices are exposed to an increased incidence of early postoperative complications and mortality. Because of significant differences in the baseline clinical characteristics of study groups, it is hard to determine whether this finding is attributable to surgical risks associated with these kinds of devices or if it is rather due to a worst pre-transplant clinical condition of MCS than nonMCS candidates. In our series, increased risk of post-transplant mortality affected patients bridged on continuous-flow and pulsatile-flow extracorporeal VADs, while post-transplant outcomes of patients bridged on VA-ECMO were similar to those of patients undergoing emergency HT under conventional therapy. Further investigation is required to clarify the optimal therapeutic pathways for HT candidates presenting with a critical clinical condition.

\section{Conflict of interest}

The authors report no relationships that could be construed as a conflict of interest.

\section{Acknowledgments}

A list of investigators and institutions that participated in this study is provided as Supplementary Data. The authors are indebted to all of them. Funding for this study was supplied by the Instituto de Salud Carlos III, Spanish Ministry of Economy and Competitiveness, through the Red de Investigación Cardiovascular. 


\section{References}

[1]. L.H. Lund, L.B. Edwards, A.Y. Kucheryavaya, et al. The Registry of the International Society for Heart and Lung Transplantation: thirtieth official adult heart transplant report-2013; focus theme: age. J Heart Lung Transplant, 32 (2013), pp. 951-964.

[2]. O. Wever-Pinzon, S.G. Drakos, A.G. Kfoury, et al. Morbidity and mortality in heart transplant candidates supported with mechanical circulatory support: is reappraisal of the current United Network for Organ Sharing thoracic organ allocation policy justified?. Circulation, 127 (2013), pp. 452-462.

[3]. J.K. Kirklin, D.C. Naftel, R.L. Kormos, et al. Fifth INTERMACS annual report: risk factor analysis from more than 6,000 mechanical circulatory support patients. J Heart Lung Transplant, 32 (2013), pp. 141-156.

[4]. A.C. Alba, M. McDonald, V. Rao, et al. The effect of ventricular assist devices on long-term post-transplant outcomes: a systematic review of observational studies. Eur J Heart Fail, 13 (2011), pp. 785-795.

[5]. J.H. Shuhaiber, K. Hur, R. Gibbons. The influence of preoperative use of ventricular assist devices on survival after heart transplantation: propensity score matched analysis. BMJ, 340 (2010), p. 392.

[6]. A.C. Alba, V. Rao, J. Ivanov, et al. Usefulness of the INTERMACS scale to predict outcomes after mechanical assist device implantation. J Heart Lung Transplant, 28 (2009), pp. 827-833.

[7]. J.F. Lazar, M.F. Swartz, M.P. Schiralli, et al. Survival after left ventricular assist device with and without temporary right ventricular support. Ann Thorac Surg, 96 (2013), pp. 2155-2159.

[8]. P.N. Mohite, B. Zych, N.R. Banner, et al. Refractory heart failure dependent on short-term mechanical circulatory support: what next? Heart transplant or long-term ventricular assist device. Artif Org, 38 (2014), pp. 276-281.

[9]. B.E. Jaski, J.C. Kim, D.C. Naftel, et al. Cardiac transplant outcome of patients supported on left ventricular assist device vs. intravenous inotropic therapy. J Heart Lung Transplant, 20 (2001), pp. 449-456.

[10]. M.A. Castel, R. Cartañá, M. Cardona, et al. Long-term outcome of high-urgency heart transplant patients with and without temporary ventricular assist device support. Transplant Proc, 44 (2012), pp. 2642-2644.

[11]. E. Barge-Caballero, J. Segovia-Cubero, L. Almenar-Bonet, et al. Preoperative INTERMACS profiles determine postoperative outcomes in critically ill patients undergoing emergency heart transplantation: analysis of the Spanish National Heart Transplant Registry. Circ Heart Fail, 6 (2013), pp. 763-772.

[12]. F. González-Vílchez, M. Gómez-Bueno, L. Almenar, et al. 24th official report of the Spanish Society of Cardiology Working Group on Heart Failure and Heart Transplantation (1984-2012). Rev Esp Cardiol, 66 (2013), pp. 973-982.

[13]. V. Patlolla, R.D. Patten, D. Denofrio, et al. The effect of ventricular assist devices on post-transplant mortality: an analysis of the United Network for Organ Sharing Thoracic Registry. J Am Coll Cardiol, 53 (2009), pp. 264271.

[14]. M. Carrier, L.P. Perrault, D. Bouchard, et al. Effect of left ventricular assist device bridging to transplantation on donor waiting time and outcomes in Canada. Can J Cardiol, 20 (2004), pp. 501-504.

[15]. E. Barth, M. Durand, C. Heylbroeck, et al. Extracorporeal life support as a bridge to high-urgency heart transplantation. Clin Transplant, 26 (2012), pp. 484-488.

[16]. T. Karamlou, J. Gelow, B.S. Diggs, et al. Mechanical circulatory support pathways that maximize post-heart transplant survival. Ann Thorac Surg, 95 (2013), pp. 480-485.

[17]. C.S. Almond, T.P. Singh, K. Gauvreau, et al. Extracorporeal membrane oxygenation for bridge to heart transplantation among children in the United States: analysis of data from the Organ Procurement and Transplant Network and Extracorporeal Life Support Organization Registry. Circulation, 123 (2011), pp. 2975-2984.

[18]. S. Shreenivas, A. Acker, P. Atluri, et al. Outcomes after ventricular assist device support in patients bridged with temporary circulatory support: analysis from INTERMACS. J Heart Lung Transplant, 32 (2013), pp. S141S142 [abstract].

[19]. M.G. Gaies, J.G. Gurney, A.H. Yen, et al. Vasoactive-inotropic score as a predictor of morbidity and mortality in infants after cardiopulmonary bypass. Pediatr Crit Care Med, 11 (2010), pp. 234-238. 